\title{
Posttraumatic Growth and Adjustment to Spinal Cord Injury: Moderated by Posttraumatic Depreciation?
}

\author{
Simon Kunz \\ Swiss Paraplegic Research (SPF) and University of Lucerne \\ Stephen Joseph \\ University of Nottingham \\ Szilvia Geyh and Claudio Peter \\ Swiss Paraplegic Research (SPF) and University of Lucerne \\ for the SwiSCI Study Group
}

Author Note

Simon Kunz, Szilvia Geyh, and Claudio Peter, Swiss Paraplegic Research (SPF), Nottwil, Switzerland and Department of Health Sciences and Health Policy, University of Lucerne, Nottwil, Switzerland; Stephen Joseph, Faculty of Social Sciences, University of Nottingham, Nottingham, UK.

This study was supported by the Swiss National Science Foundation (Grant 100014_149284). This study has been financed in the framework of the Swiss Spinal Cord Injury Cohort Study (SwiSCI, www.swisci.ch ), supported by the Swiss Paraplegic Foundation. The members of the SwiSCI Steering Committee are: Xavier Jordan, Bertrand Léger (Clinique Romande de Réadaptation, Sion); Michael Baumberger, Hans Peter Gmünder (Swiss Paraplegic Center, Nottwil); Armin Curt, Martin Schubert (University Clinic Balgrist, Zürich); Margret Hund-Georgiadis, Kerstin Hug (REHAB Basel, Basel); Hans Georg Koch, (Swiss Paraplegic Association, Nottwil); Hardy Landolt (Representative of persons with SCI, Glarus); Nadja Münzel (Parahelp, Nottwil); Mirjam Brach, Gerold Stucki (Swiss Paraplegic Research, Nottwil); Martin Brinkhof, Christine Thyrian (SwiSCI Study Center at Swiss Paraplegic Research, Nottwil). We thank Dr. Christina Werner (University of Zurich, Switzerland) for her advising in statistical analyses.

Correspondence concerning this article should be addressed to Simon Kunz, Swiss Paraplegic Research (SPF), Guido A. Zäch-Strasse 4, CH-6207 Nottwil, Switzerland. E-mail: simon.kunz@ paraplegie.ch 


\begin{abstract}
Objective: Findings on the relationship of posttraumatic growth (PTG) with adjustment to potentially traumatic events are inconsistent, whereupon posttraumatic depreciation (PTD) has been suggested as a possible moderator. The objective of this study is to investigate the associations between PTG and PTD on one side and life satisfaction and indicators of mental and physical health on the other side in individuals with spinal cord injury (SCI). The primary study aim is to test whether PTD moderates the relationships of PTG and different adjustment indicators. Method: A total of 141 patients administered to one of four Swiss SCI rehabilitation centers completed questionnaires assessing PTG and PTD, different indicators of mental and physical health as well as life satisfaction at discharge from first rehabilitation. Correlational and regression methods were used to examine the research question. Results: PTG and PTD were significantly positively correlated $\left(r_{s}=.47\right)$. PTD was significantly associated with lower mental and physical health and lower life satisfaction, with small to large effect sizes. PTD moderated the associations of PTG with symptoms of depression and life satisfaction ( $\beta$ of interaction term $=-.18$ and .24 , respectively). PTG was significantly related to lower levels of symptoms of depression and higher life satisfaction in individuals experiencing moderate to high levels of PTD. In contrast, PTG was not significantly related to these outcomes in individuals with low PTD levels. Conclusion: The neglect of PTD in research partially explains mixed findings on the relationship of PTG and adjustment to potentially traumatic events.
\end{abstract}

Keywords: spinal cord injury, posttraumatic growth, quality of life, depression, psychological adjustment 
Spinal cord injury (SCI) is a life changing event which leads to chronic disability and confronts the affected individual with severe physical, social and psychological challenges (W.H.O., 2013). Individuals with SCI are at risk for elevated symptoms of depression, anxiety, and global distress or having a reduced quality of life (Post \& van Leeuwen, 2012).

Despite this potential negative impact, a growing amount of studies demonstrate that individuals with SCI also perceive positive psychological changes following the injury (e.g., January, Zebracki, Chlan, \& Vogel, 2015; Kennedy, Lude, Elfström, \& Cox, 2013). Salient domains are, for example, better relationships with others, new goals or priorities, or a greater appreciation of life (Kennedy et al., 2013). They are subsumed under the term posttraumatic growth (PTG), which includes "positive psychological changes experienced as a result of the struggle with highly challenging life circumstances" (Tedeschi \& Calhoun, 2004, p. 1).

\section{Posttraumatic Growth and Adjustment to Potentially Traumatic Events}

An open question in research on PTG after potentially traumatic events such as SCI is whether PTG is associated with better (mental) health and hence should be considered as a primary intervention target in clinical research and practice (Zoellner \& Maercker, 2006). In this respect, several systematic literature reviews reported mixed findings regarding the relationship of PTG with different indicators of physical and mental health in individuals who experienced potentially traumatic events (e.g., Helgeson, Reynolds, \& Tomich, 2006; Sawyer, Ayers, \& Field, 2010; Shand, Cowlishaw, Brooker, Burney, \& Ricciardelli, 2015; Zoellner \& Maercker, 2006). PTG research in individuals with SCI is sparse (Kennedy et al., 2013), but mirrors these inconsistent findings. For example, whereas some studies found PTG to be negatively related to symptoms of posttraumatic stress (Elfström, Kreuter, Ryden, Persson, \& Sullivan, 2002) and depression (Min et al., 2014), others found no significant associations (Kalpakjian et al., 2014; McMillen \& Cook, 2003).

As possible explanations for these inconsistent findings, demographic and trauma- 
related characteristics were suggested and tested as potential moderators of the relationship between PTG and adjustment indicators in meta-analyses (e.g., Helgeson et al., 2006; Sawyer et al., 2010). However, substantial unexplained variability remained in the effect sizes across different studies and the need to identify other potential moderators in empirical studies was stressed (Sawyer et al., 2010). Negative psychological consequences of potentially traumatic events may have such a moderating function.

PTG research has predominantly used measures enabling respondents to report only positive psychological changes (Park \& Lechner, 2006) and neglected that negative changes can arise in the same domains as PTG. These negative changes have been termed posttraumatic depreciation (PTD; Baker, Kelly, Calhoun, Cann, \& Tedeschi, 2008) and have not been examined in individuals with SCI so far. However, individuals who experienced other potentially traumatic events reported PTD independent of and in lower levels than PTG, with PTD being negatively associated with life satisfaction and indicators of mental health (Barrington \& Shakespeare-Finch, 2013; Cann, Calhoun, Tedeschi, \& Solomon, 2010).

So far, only one study examined the potential moderating effect of PTD on the relationship of PTG with indicators of adjustment. Cann, Calhoun, Tedeschi, and Solomon (2010) found PTD to moderate the relationship of PTG with life satisfaction and presence of meaning in life. In individuals experiencing low levels of PTD, PTG was not significantly related to life satisfaction and presence of meaning in life. In contrast, among the individuals with high levels of PTD, PTG was positively related to life satisfaction and meaning in life.

This suggests that PTG may act as a stress buffer, i.e. a variable related to adjustment only when a potentially traumatic event has (severe) negative consequences for the affected individual (e.g., Silva, Moreira, \& Canavarro, 2012). It seems plausible to assume that under such circumstances PTG is not only related to more meaning in and satisfaction with life but also to less distress and dysfunction by enabling to focus not entirely on the negative 
consequences but also on positive ones. To date, such an interaction effect of PTG and PTD regarding distress and dysfunction has not been examined. Nonetheless, if negative consequences of a potentially traumatic event were operationalized differently, e.g. with the perceived overall negative impact of a chronic illness (Silva et al., 2012), an interaction effect was also found regarding symptoms of depression, but not regarding symptoms of anxiety and physical health, which seemed hardly associated with PTG at all (e.g., Bluvstein, Moravchick, Sheps, Schreiber, \& Bloch, 2013; Morrill et al., 2008; Silva et al., 2012).

\section{Current Study}

To conclude, the association of PTG with adjustment to SCI and other potentially traumatic events is unclear, but may depend on the amount of negative consequences perceived after the event. In this regard, the role of PTD needs further examination. Consequently, the objective of this study is to investigate the associations between PTG and PTD on one side and life satisfaction and indicators of mental (symptoms of depression, anxiety, global distress) and physical health (physical functioning, pain) on the other side in individuals with SCI at rehabilitation discharge. More specifically, the study aims are (a) to investigate whether individuals with SCI perceive PTG and PTD, (b) to examine how PTD is related to PTG and to different indicators of life satisfaction, mental health, and physical health, and (c) to test if PTD moderates the relationships of PTG with these different adjustment indicators. Based on the literature outlined above, we expect PTG and PTD to be reported, but PTG more often and to a higher degree than PTD (aim a). We also hypothesize that PTD is unrelated to PTG and physical health, but negatively associated with adjustment across different indicators of mental health and life satisfaction (aim b). Finally, we expect PTD to moderate the associations between PTG and symptoms of depression, life satisfaction, and global distress: We predict that PTG is not significantly related to these outcomes in individuals with low PTD levels, but shows a positive association with life 
satisfaction and a negative association with symptoms of depression and global distress in individuals with higher PTD levels. Independent of PTD levels, we expect PTG not to be related to symptoms of anxiety and physical health (aim c).

\section{Method}

\section{Participants and Procedure}

Participants in the current study were recruited in the Swiss Spinal Cord Injury Cohort Study (SwiSCI) in pathway 3 (Post et al., 2011). Performed by the Swiss Paraplegic Research, SwiSCI pathway 3 is a prospective multi-center longitudinal survey, which aims to describe individuals living with SCI in various dimensions such as health maintenance or quality of life. It includes individuals who are newly diagnosed with traumatic or nontraumatic SCI and who are admitted for first rehabilitation in one of the four Swiss rehabilitation centers (Spinal Cord Injury Center of the Balgrist University Hospital, Zürich; Centre for Spinal Cord Injury and Severe Head Injury, REHAB Basel; Clinique Romande de Réadaptation, Sion; and the Swiss Paraplegic Centre, Nottwil). Further inclusion criteria are that the person is aged 16 years or older, and has a permanent residence in Switzerland. Individuals with the following criteria are excluded: congenital conditions leading to paraplegia or tetraplegia, including spina bifida, new SCI in the context of palliative (end-oflife) care, and neurodegenerative disorders such as multiple sclerosis.

The cantonal ethics committees approved SwiSCI and all participants give informed consent. SwiSCI pathway 3 data are collected prospectively at one, three, and six months after the diagnosis of the spinal cord lesion, at discharge from first rehabilitation, and two and five years after the diagnosis (Post et al., 2011). However, duration of first rehabilitation varies (see Table 1), depending, for example, on the severity of the injury. Some participants are discharged earlier and consequently not every participant completes all the envisioned 
SwiSCI measurement occasions prior to the discharge assessment.

The current study used cross-sectional data assessed at discharge from first rehabilitation (Time 4). Its reporting is based on the "Strengthening the reporting of observational studies in epidemiology (STROBE)" statement. The sample included all participants who completed Time 4 until August $10^{\text {th }} 2015$. Of these, eight participants were excluded, because they did not answer the questions assessing the main variables of interest, PTG and PTD. The resulting sample size was $N=141$. Demographic and injury-related characteristics are summarized in Table 1.

[Insert Table 1 around here]

\section{Measures}

Mental health. The global distress resulting from SCI was measured using the oneitem Distress Thermometer (DT; Roth et al., 1998). Participants rated their currently experienced level of distress on a scale from 0 (= no distress) to 10 (= very high distress). The DT is a widely used and extensively validated screening instrument to assess distress caused by specific health conditions (Snowden et al., 2011). The recommended cut-off indicating clinically relevant levels of global distress is a score of 4 (Snowden et al., 2011). Symptoms of depression and anxiety were assessed by using the Hospital Anxiety and Depression Scale (HADS; Zigmond \& Snaith, 1983). The HADS was constructed to screen for clinically relevant levels of symptoms of depression and anxiety in patients attending a medical clinic. It contains 14 items in two subscales with seven items each assessing symptoms of depression and anxiety. Patients rated their feelings during the past week on a Likert scale ranging from 0 to 3 . Higher scores imply higher levels of symptoms of depression and anxiety, with subscale values between 8-10 indicating borderline and values starting from 11 indicating clinically relevant levels of symptoms of depression and anxiety. The HADS is a reliable and valid measure in individuals with SCI and in other clinical 
populations (Bjelland, Dahl, Haug, \& Neckelmann, 2002; Müller, Cieza, \& Geyh, 2012).

Life satisfaction. Two measures were used to collect the participant's life satisfaction. First, five selected items of the World Health Organization Quality of Life Assessment (WHOQoL; Geyh, Fellinghauer, Kirchberger, \& Post, 2010) were used. Participants rated their overall life satisfaction as well as their satisfaction with specific life domains (e.g. health or living conditions) on a five-point Likert scale from 1 to 5. Item scores are summed up to calculate a total score. The five selected items of the WHOQoL were shown to be a reliable and valid measure in SCI samples (Geyh et al., 2010). The second measure was the International Spinal Cord Injury Quality of Life Basic Data Set (SCI QoL Basic Data Set; Charlifue et al., 2012). Participants rated their general life satisfaction as well as their satisfaction with physical and psychological health in three items ranging from 0 (= completely dissatisfied $)$ to 10 (= completely satisfied $)$. A mean score is calculated across these items. The SCI QoL Basic Data Set showed good internal consistency, construct and convergent validity (Post, Adriaansen, Charlifue, Biering-Sorensen, \& van Asbeck, 2015).

Physical health. Physical functioning was measured using the Spinal Cord Independence Measure version III (SCIM III; Catz et al., 2007). The SCIM III contains 19 items on different daily tasks (e.g. transfer from bed to wheelchair, grooming). Health practitioners rated the performance of the participants. According to a scoring algorithm, a total score is calculated. The SCIM III proved to be reliable and valid (Davis, NolenHoeksema, \& Larson, 1998). Pain intensity, a frequent secondary complication of SCI (Ehde et al., 2003), was measured with one item from the International Spinal Cord Injury Basic Pain Data Set (ISCIBPDS; Widerstrom-Noga et al., 2008). Participants rated the average pain intensity in the past week on a scale from $0(=$ no pain at all $)$ to 10 (=worst pain to imagine $)$.

PTG and PTD. PTG was measured using the short version of the Posttraumatic Growth Inventory (PTGI-SF; Cann, Calhoun, Tedeschi, Taku, et al., 2010). The PTGI-SF 
includes 10 items regarding positive changes after a potentially traumatic event. Participants indicated the degree to which they experienced the respective change in each item using a six-point Likert scale from 0 (=I did not experience this change) to 5 (=I experienced this change to a very great degree). The PTGI-SF has five subscales composed of two items representing growth in the domains improved relationships, recognizing new possibilities for one's life, a greater appreciation of life, a greater sense of personal strength, and spiritual development. PTD was measured selecting the 10 corresponding but negatively worded items from the Paired Format Posttraumatic Growth Inventory (PTGI-42; Baker et al., 2008). Both, the PTGI-SF and the PTGI-42 demonstrated to be reliable and valid instruments (Baker et al., 2008; Cann, Calhoun, Tedeschi, Taku, et al., 2010). However, because the reduced 20 item version has not been used so far, a confirmatory factor analysis was conducted using the lavaan package in R (Rosseel, 2012). A hierarchical model with two higher order factors (PTG and PTD) and each with five lower order factors representing the subscales was tested. Using robust maximum likelihood estimation, this model reached an acceptable fit. The $\chi^{2}$ test of the model fit was significant $\left(\chi^{2}=227.75, d f=161, p<.001\right)$, the comparative fit index (CFI) was .93, and the root-mean square error of approximation (RMSEA) was .05. All items had substantial loadings on the respective subscale ( $\beta$ between .60 and .83 ) and all of the subscales on the respective higher order factors ( $\beta$ between .59 and 1.00).

\section{Results}

Prior to the main analyses, missing values were imputed using the missForest package in R (Stekhoven \& Buhlmann, 2012). If not further specified below, the following analyses were conducted using SPSS, version 18.

\section{Perceptions of PTG and PTD}

In order to investigate whether both PTG and PTD were perceived and to examine the 
distributions of the adjustment indicators, descriptive statistics were calculated (Table 2). As can be seen in Table 2, only 3 participants indicated to experience PTG not at all. This means that $97.9 \%$ of the participants indicated to have experienced PTG to some degree. In contrast, only $83.7 \%$ reported PTD. On the subscale level, only a minority of participants reported positive $(44.7 \%)$ or negative $(29.8 \%)$ changes in spirituality. Regarding the other subscales, 2 to 4 times as many participants reported to experience positive compared to negative changes.

[Insert Table 2 around here]

The mean values of PTG and PTD total and subscale scores were compared by Wilcoxon signed-rank tests, because the Shapiro-Wilk test indicated nonnormal distributions of these variables (Table 2). The comparison between the total scores revealed a significantly higher PTG score $(Z=8.396, p<.001, r=.71)$. In addition, all PTG subscale scores were significantly higher than the corresponding PTD scores. Following Cohen (1988), effect sizes ranged from a small effect between the spiritual change scales $(Z=3.373, p<.001, r=.28)$ to a large effect between the relating to others scales $(Z=7.132, p<.001, r=.60)$.

\section{Bivariate Correlations Between PTG, PTD, and the Adjustment Indicators}

According to the nonnormal distributions, bivariate Spearman correlations between PTG and PTD total scores, the subscales scores and the adjustment indicators were calculated (Table 3). PTG and PTD total scores $\left(r_{s}=.47, p<.001\right)$ as well as most of the subscales were significantly positively associated. Regarding the subscales, the smallest effect size was $r_{s}=$ $.11, p=.190$ between the PTG relating to others and the PTD appreciation of life subscales. The largest effect size was $r_{s}=.43, p<.001$ between the PTG new possibilities and the PTD relating to others subscales. Most of the observed effect sizes were moderate (Cohen, 1988).

[Insert Table 3 around here]

With respect to the adjustment indicators, the PTG total score was significantly related to global distress $\left(r_{s}=.24, p=.005\right)$ and symptoms of anxiety $\left(r_{s}=.28, p<.001\right)$. 
These effects were weak. The PTD total score was significantly negatively related to satisfaction with life, and all indicators of mental and physical health. Effect sizes were moderate to strong with exception of those between the PTD total score and pain intensity $\left(r_{s}\right.$ $=.26, p=.002)$ as well as physical functioning $\left(r_{s}=-.26, p=.002\right)$, which were small.

\section{Moderator Analyses}

To explore the hypothesized interaction effect of PTD and PTG in predicting the adjustment indicators, we conducted a series of hierarchical regression analyses. In each regression model, one of the adjustment indicators was the dependent variable and the centered PTG and PTD total scores were blocked and entered in a first step as independent variables (Model 1). In a second step, their interaction was included as a third independent variable (Model 2). To determine the effect size of the interaction, the variation of Cohen's $f^{2}$ measuring local effect size was calculated (Table 4).

As can be seen in Table 4, PTD was significantly associated with all adjustment indicators, with standardized regression coefficients in Model 1 ranging from .22 to .66. PTG was significantly associated with symptoms of depression $(\beta=-.20, p=.012)$ and life satisfaction (selected WHOQoL items: $\beta=.22, p=.009$; SCI QoL Basic Data Set: $\beta=.19, p$ $=.029)$. The interaction term was significant in the regression models regarding symptoms of depression, life satisfaction, and pain intensity. With an $f^{2}$ between .04 and .06 , all of these significant effects were weak (see Cohen, 1988). PTG, PTD, and their interaction term explained between $6 \%$ and $39 \%$ of the variance in the adjustment indicators in Models 2.

[Insert Table 4 around here]

The significant interaction terms were probed by graphing the relation of PTG with the adjustment indicator for participants at the mean and for those at $\pm 1 S D$ from the mean of PTD (Figure 1). Following Bauer and Curran (2005), we also tested whether these depicted simple slopes were significantly different from zero and over what range of PTD scores PTG 
had a significant association with the respective adjustment indicator (region of significance). To do so, computational tools created by Preacher, Curran, and Bauer (2006) were used. With respect to symptoms of depression, the simple slopes shown in Figure 1a were not significant for participants at $1 S D$ below the mean of centered PTD $(B=-0.023, p=$ $.481)$, but for participants at the mean $(B=-.075, p=.003)$, and $1 S D$ above the mean $(B=-$ $0.127, p \leq .001)$ the slope was significant, indicating a negative association between PTG and symptoms of depression. The region of significance indicated that simple slopes were significant for all participants with a centered PTD total score larger than -4.494 .

[Insert Figure 1 around here]

Concerning the interactions in the regressions on the life satisfaction measures (see Figure $1 \mathrm{~b}$ and $1 \mathrm{c})$, the simple slopes were not significant for participants reporting PTD scores at $1 S D$ below the mean in regard to the selected WHOQoL items $(B=0.014, p=$ $.670)$ and to the SCI QoL Basic Data Set $(B=-0.003, p=.881)$. For participants at the mean of PTD (selected WHOQoL items: $B=0.076, p=.002$; SCI QoL Basic Data Set: $B=0.039$, $p=.006$ ) and $1 S D$ above the mean (selected WHOQoL items: $B=0.138, p \leq .001$; SCI QoL Basic Data Set: $B=0.081, p \leq .001$ ) the slopes were significant, indicating a positive association between PTG and life satisfaction. According to the regions of significance, the slopes were significant at centered PTD values of -4.271 and larger regarding the selected WHOQoL items and at -2.701 and larger regarding the SCI QoL Basic Data Set.

Concerning pain intensity, none of the slopes in Figure 1d reached statistical significance. Regions of significance indicate that only slopes at centered PTD smaller than $15.630(B=.079, p=.05)$ and larger than $16.701(B=-.083, p=.05)$ reached significance.

\section{Sensitivity Analyses}

First, to check for potential bias resulting from imputation, all analyses were rerun with cases with more than $20 \%$ missing values in the questionnaire items excluded. No 
discrepancies were detected. Second, to check whether possible violations of the underlying assumptions, as indicated by visual inspection of the residual distributions, led to biased results, regression analyses were rerun using nonparametric bootstrapping with 5000 repetitions in Stata, version 13. Again, no discrepancies were detected. Third, the data was inspected for potential predictor outliers and potential influential cases based on cut-off scores in centered leverage and standardized DFFit recommended by Aguinis, Gottfredson, and Joo (2013). Four potential predictor outliers and between five (global distress) and twelve (symptoms of anxiety) potential influential cases were identified. The regression analyses were rerun with these cases excluded. When the potential influential cases were excluded, no discrepancies were detected. The only discrepancy when the potential predictor outliers were excluded was that interaction term of PTG and PTD lost its significance in the regression model with regard to symptoms of depression $(\beta=-.12, p=.138)$ and pain intensity $(\beta=-.10$, $p=.266)$. However, all of the potentially outlying cases were considered as belonging to the examined population and thus only results with these cases included are reported.

\section{Discussion}

In line with research after other potentially traumatic events, the current study found both PTG and PTD to be reported in individuals with SCI at rehabilitation discharge, but PTG more frequently and to a higher degree than PTD. Our hypotheses regarding the associations of PTD with adjustment were partially supported: As expected, PTD was associated with lower life satisfaction and lower mental health. Contrary to our expectations, we also found PTD to be positively related to PTG and negatively related to physical health (physical functioning and pain intensity). Our hypotheses regarding the moderating effect of PTD on the relationship of PTG with adjustment were also partially supported: As expected, PTD moderated the effects of PTG with symptoms of depression and life satisfaction. 
Contrary to our expectations, PTD also moderated the relationship of PTG with pain intensity, but not with global distress.

A possible explanation for the strong positive correlation between PTG and PTD might be that the potentially traumatic event was relatively recent for most individuals (mean of 5.4 months). Joseph et al. (2005) argued that the initial time after trauma may be characterized by a disorganization of schematic structures and that reorganization through cognitive and emotional processing takes time. Therefore, PTG and PTD following trauma may be positively related initially after trauma, but they become increasingly dissociated over time when individuals are able to reorganize the trauma-related information in a meaningful way. As an exploratory test of this argument, we did an extreme group analysis. We split the participants of the current study into four equal groups (quartiles) differing in time since injury and compared the correlation in the lower and the upper quartile. Corroborating our argument, we found a strong positive correlation $\left(r_{s}=.59, p<.001\right)$ in the lower quartile (range of time since injury $=27-84$ days $)$ and a considerably smaller correlation $\left(r_{s}=.29, p=\right.$ .09 ) in the upper quartile (range of time since injury $=224-485$ days). ${ }^{1}$ Nevertheless, longitudinal studies specifically designed to examine the relationship of PTG and PTD in the same sample at several points in a longer period of time are needed for clarification.

The finding that higher PTD was consistently associated with lower mental health and lower life satisfaction was also observed in individuals after other potentially traumatic events (e.g., Barrington \& Shakespeare-Finch, 2013). Interestingly, we also found higher reports of PTD to be associated with worse physical functioning and higher pain intensity.

${ }^{1}$ Because time since injury may be a proxy for injury severity, a reviewer suggested to check for severity as potential moderator of the PTG-PTD association. Post-hoc analyses revealed no substantial differences $\left(\Delta r_{s}<.07\right)$ across groups differing in injury severity. 
Regarding physical functioning, the PTD domains less possibilities in life, personal strength, and appreciation of life showed the strongest associations. Thus, actual barriers may be mirrored in these perceptions of PTD. For example, individuals with low preserved physical functioning after SCI are more limited in leisure and professional activities and hence may perceive fewer possibilities in life. Pain, on the other hand, is frequently accompanied by an increased tendency in catastrophic cognitions in individuals with SCI during first rehabilitation (Nicholson Perry, Nicholas, \& Middleton, 2009). Therefore, individuals with high levels of pain might be especially prone to detect and focus on PTD.

Of major interest for the current study was whether PTD moderates the relationship between PTG and adjustment indicators. We found PTG not to be associated with symptoms of depression and life satisfaction when individuals perceived comparatively low levels of PTD. In contrast, at moderate and high levels of PTD, PTG was significantly related to lower levels of symptoms of depression and more life satisfaction. These results may be seen as support for the notion that PTG can have an adaptive, stress buffering function especially when concurrent negative life changes are not denied (e.g., Zoellner \& Maercker, 2006).

The results may also be seen as support for the PTG model of Tedeschi and Calhoun (2004), which assumes real PTG to be experienced only after potentially traumatic events which are subjectively challenging or impactful. Similar to the findings in the current study, a recent study by Johnson and Boals (2015) demonstrated that retrospective self-reports of PTG were only positively related to adjustment when the individuals perceived the event as having had a high impact on their identity. Moreover, in contrast to individuals less impacted by the event, these individuals seemed to have "realistic perceptions" of PTG, as their reports more accurately reflected longitudinal changes in PTG domains. This suggests that perceptions of PTG may only be adaptive when representing real changes. Considering the significant moderation effects in the current study, acknowledging PTD (i.e. negative impact of the 
event) besides PTG could indicate such realistic perceptions of posttraumatic life changes (see also Cheng, Wong, \& Tsang, 2006, for a similar discussion).

However, our results indicate that the adaptive function of PTG is limited. We found neither significant main effects of PTG nor a significant interaction with PTD regarding symptoms of anxiety and global distress. Likewise, Silva et al. (2012) found PTG not to be associated with symptoms of anxiety even when a possible interaction with the global negative impact of trauma was taken into account. Examining individuals undergoing cancer treatment, Silva et al. argued that anxiety (and similarly global distress) resulting from a health-related trauma is not easily changeable by the experience of PTG, but rather is influenced by the circumstances affected individuals currently have to deal with. In the case of individuals with SCI at rehabilitation discharge, this might include uncertainty about the vocational future as well as other challenges associated with returning to the community (see W.H.O., 2013, for an overview).

Similarly, we found PTG to be unrelated to physical functioning and, for most of the individuals, pain intensity independent of PTD levels. Only for individuals very high and very low in PTD (more than $\pm 1 S D$ from the mean) PTG was significantly related to pain intensity. For those very high in PTD, this association was negative. One possible explanation might be that these individuals were also high in catastrophic cognitions. Such negative mental sets can interfere with the experience of PTG as evidence from a sample of breast cancer survivors illustrated (Porter et al., 2006). The finding that PTD was positively correlated with pain intensity, which in turn is associated with such negative mental sets, as argued above, seems to further support this claim. In contrast, PTG was positively associated with pain intensity for individuals very low in PTD. It might be that, as indicated by the low PTD levels, these individuals struggled less with SCI. As argued above, according to the PTG model of Tedeschi and Calhoun (2004), it is the struggle with such consequences rather than 
trauma itself which stimulates PTG. Therefore it might be that struggles resulting from the experience of pain rather than SCI stimulated PTG in these individuals.

The current study is subject to limitations. First, causal interpretations such as above remain speculations because the current study had a cross-sectional design. Second, the interaction effects of PTG and PTD with respect to depression and pain intensity were not only weak but also unstable. Estimating the minimally required sample size to detect interaction effects in ordinary least squares regression models can hardly be determined (Dawson, 2014). Thus, we based our expectation on the sample size of other studies finding PTG to interact with negative consequences of trauma (e.g., Cann, Calhoun, Tedeschi, \& Solomon, 2010). Nevertheless, the instability of the interaction effects when few extreme cases were excluded indicates the need to replicate the current study in a larger sample.

To conclude, the neglect of PTD may partially explain mixed findings in research on the relationship of PTG and adjustment to potentially traumatic events. The current research provided further insights into the potential moderating role of PTD: PTG was only related to better adjustment in individuals experiencing also higher levels of PTD. However, whether PTG perceived initially after trauma is related to better adjustment in the longer run remains unclear, as it might reflect illusory, self-deceptive change (e.g., Zoellner \& Maercker, 2006). A next step to answer the question regarding the significance of PTG in clinical practice would be to examine the adaptive effect of PTG in individuals reporting higher and lower levels of PTD in longitudinal studies. Similarly, whether PTD is related to worse adjustment later on has neither been examined so far. It may be that PTD is associated with more psychological discomfort in cross-sectional designs, but as it could represent a realistic view on posttraumatic transformation, it may not necessarily be related to worse adjustment over time. Nevertheless, the results of the current study indicate that fostering the experience of PTG in clinical interventions should be targeted especially in individuals experiencing PTD. 


\section{References}

Aguinis, H., Gottfredson, R. K., \& Joo, H. (2013). Best-practice recommendations for defining, identifying, and handling outliers. Organizational Research Methods, 16, 270-301. doi: 10.1177/1094428112470848

Baker, J. M., Kelly, C., Calhoun, L. G., Cann, A., \& Tedeschi, R. G. (2008). An examination of posttraumatic growth and posttraumatic depreciation: Two exploratory studies. Journal of Loss and Trauma, 13, 450-465. doi: 10.1080/15325020802171367

Barrington, A., \& Shakespeare-Finch, J. (2013). Posttraumatic growth and posttraumatic depreciation as predictors of psychological adjustment. Journal of Loss and Trauma, 18, 429-443. doi: 10.1080/15325024.2012.714210

Bauer, D. J., \& Curran, P. J. (2005). Probing interactions in fixed and multilevel regression: Inferential and graphical techniques. Multivariate Behavioral Research, 40, 373-400. doi: 10.1207/s15327906mbr4003_5

Bjelland, I., Dahl, A. A., Haug, T. T., \& Neckelmann, D. (2002). The validity of the Hospital Anxiety and Depression Scale: An updated literature review. Journal of Psychosomatic Research, 52, 69-77. doi: 10.1016/s0022-3999(01)00296-3

Bluvstein, I., Moravchick, L., Sheps, D., Schreiber, S., \& Bloch, M. (2013). Posttraumatic growth, posttraumatic stress symptoms and mental health among coronary heart disease survivors. Journal of Clinical Psychology in Medical Settings, 20, 164-172. doi: 10.1007/s10880-012-9318-z

Cann, A., Calhoun, L. G., Tedeschi, R. G., \& Solomon, D. T. (2010). Posttraumatic growth and depreciation as independent experiences and predictors of well-being. Journal of Loss and Trauma, 15, 151-166. doi: 10.1080/15325020903375826

Cann, A., Calhoun, L. G., Tedeschi, R. G., Taku, K., Vishnevsky, T., Triplett, K. N., \& Danhauer, S. C. (2010). A short form of the Posttraumatic Growth Inventory. Anxiety, 
Stress, \& Coping: An International Journal, 23, 127-137. doi: $10.1080 / 10615800903094273$

Catz, A., Itzkovich, M., Tesio, L., Biering-Sorensen, F., Weeks, C., Laramee, M. T., . . . Ronen, J. (2007). A multicenter international study on the Spinal Cord Independence Measure, version III: Rasch psychometric validation. Spinal Cord, 45, 275-291. doi: 10.1038/sj.sc.3101960

Charlifue, S., Post, M. W. M., Biering-Sorensen, F., Catz, A., Dijkers, M., Geyh, S., ... Sinnott, K. A. (2012). International Spinal Cord Injury Quality of Life Basic Data Set. Spinal Cord, 50, 672-675. doi: 10.1038/sc.2012.27

Cheng, C., Wong, W. M., \& Tsang, K. W. (2006). Perception of benefits and costs during SARS outbreak: An 18-month prospective study. Journal of Consulting and Clinical Psychology, 74, 870-879. doi: 10.1037/0022-006x.74.5.870

Cohen, J. (1988). Statistical power analysis for the behavioral sciences (2nd ed.). Hillsdale, MI: Lawrence Erlbaum.

Davis, C. G., Nolen-Hoeksema, S., \& Larson, J. (1998). Making sense of loss and benefiting from the experience: Two construals of meaning. Journal of Personality and Social Psychology, 75, 561-574. doi: 10.1037/0022-3514.75.2.561

Dawson, J. F. (2014). Moderation in management research: What, why, when, and how. Journal of Business and Psychology, 29, 1-19. doi: 10.1007/s10869-013-9308-7

Ehde, D. M., Jensen, M. P., Engel, J. M., Turner, J. A., Hoffman, A. J., \& Cardenas, D. D. (2003). Chronic pain secondary to disability: A review. The Clinical Journal of Pain, 19, 3-17. doi: 10.1097/00002508-200301000-00002

Elfström, M. L., Kreuter, M., Ryden, A., Persson, L. O., \& Sullivan, M. (2002). Effects of coping on psychological outcome when controlling for background variables: A study of traumatically spinal cord lesioned persons. Spinal Cord, 40, 408-415. doi: 
10.1038/sj.sc.3101299

Geyh, S., Fellinghauer, B. A., Kirchberger, I., \& Post, M. W. M. (2010). Cross-cultural validity of four quality of life scales in persons with spinal cord injury. Health and Quality of Life Outcomes, 8, 94. doi: 10.1186/1477-7525-8-94

Helgeson, V. S., Reynolds, K. A., \& Tomich, P. L. (2006). A meta-analytic review of benefit finding and growth. Journal of Consulting and Clinical Psychology, 74, 797-816. doi: 10.1037/0022-006x.74.5.797

January, A. M., Zebracki, K., Chlan, K. M., \& Vogel, L. C. (2015). Understanding posttraumatic growth following pediatric-onset spinal cord injury: The critical role of coping strategies for facilitating positive psychological outcomes. Developmental Medicine \& Child Neurology, 57, 1143-1149. doi: 10.1111/dmcn.12820

Johnson, S. F., \& Boals, A. (2015). Refining our ability to measure posttraumatic growth. Psychological Trauma: Theory, Research, Practice, and Policy, 7, 422-429. doi: $10.1037 / \operatorname{tra} 0000013$

Joseph, S., Linley, P. A., Andrews, L., Harris, G., Howle, B., Woodward, C., \& Shevlin, M. (2005). Assessing positive and negative changes in the aftermath of adversity: Psychometric evaluation of the Changes In Outlook Questionnaire. Psychological Assessment, 17, 70-80. doi: 10.1037/1040-3590.17.1.70

Kalpakjian, C. Z., McCullumsmith, C. B., Fann, J. R., Richards, J. S., Stoelb, B. L., Heinemann, A. W., \& Bombardier, C. H. (2014). Post-traumatic growth following spinal cord injury. The Journal of Spinal Cord Medicine, 37, 218-225. doi: $10.1179 / 2045772313 Y .0000000169$

Kennedy, P., Lude, P., Elfström, M. L., \& Cox, A. (2013). Perceptions of gain following spinal cord injury: A qualitative analysis. Topics in Spinal Cord Injury Rehabilitation, 19, 202-210. doi: 10.1310/sci1903-202 
McMillen, J. C., \& Cook, C. L. (2003). The positive by-products of spinal cord injury and their correlates. Rehabilitation Psychology, 48, 77-85. doi: 10.1037/00905550.48 .2 .77

Min, J. A., Lee, C. U., Hwang, S. I., Shin, J. I., Lee, B. S., Han, S. H., . . Chae, J. H. (2014). The moderation of resilience on the negative effect of pain on depression and posttraumatic growth in individuals with spinal cord injury. Disability and Rehabilitation, 36, 1196-1202. doi: 10.3109/09638288.2013.834985

Morrill, E. F., Brewer, N. T., O'Neill, S. C., Lillie, S. E., Dees, E. C., Carey, L. A., \& Rimer, B. K. (2008). The interaction of post-traumatic growth and post-traumatic stress symptoms in predicting depressive symptoms and quality of life. Psycho-Oncology, 17, 948-953. doi: 10.1002/pon.1313

Müller, R., Cieza, A., \& Geyh, S. (2012). Rasch analysis of the Hospital Anxiety and Depression Scale in spinal cord injury. Rehabilitation Psychology, 57, 214-223. doi: $10.1037 / \mathrm{a} 0029287$

Nicholson Perry, K., Nicholas, M. K., \& Middleton, J. (2009). Spinal cord injury-related pain in rehabilitation: A cross-sectional study of relationships with cognitions, mood and physical function. European Journal of Pain, 13, 511-517. doi: 10.1016/j.ejpain.2008.06.003

Park, C. L., \& Lechner, S. C. (2006). Measurement issues in assessing growth following stressful life experiences. In L. G. Calhoun \& R. G. Tedeschi (Eds.), Handbook of posttraumatic growth: Research and practice (pp. 47-67). Mahwah, NJ: Lawrence Erlbaum.

Porter, L. S., Clayton, M. F., Belyea, M., Mishel, M., Gil, K. M., \& Germino, B. B. (2006). Predicting negative mood state and personal growth in African American and White long-term breast cancer survivors. Annals of Behavioral Medicine, 31, 195-204. doi: 
10.1207/s15324796abm3103_1

Post, M. W. M., Adriaansen, J. J., Charlifue, S., Biering-Sorensen, F., \& van Asbeck, F. W. (2015). Good validity of the International Spinal Cord Injury Quality of Life Basic Data Set. Spinal Cord, Advance online publication. doi: 10.1038/sc.2015.99

Post, M. W. M., Brinkhof, M. W., von Elm, E., Boldt, C., Brach, M., Fekete, C., . . Stucki, G. (2011). Design of the Swiss Spinal Cord Injury Cohort Study. American Journal of Physical Medicine and Rehabilitation, 90(Suppl. 2), 5-16. doi: 10.1097/PHM.0b013e318230fd41

Post, M. W. M., \& van Leeuwen, C. M. C. (2012). Psychosocial issues in spinal cord injury: A review. Spinal Cord, 50, 382-389. doi: 10.1038/sc.2011.182

Preacher, K. J., Curran, P. J., \& Bauer, D. J. (2006). Computational tools for probing interaction effects in multiple linear regression, multilevel modeling, and latent curve analysis. Journal of Educational and Behavioral Statistics, 31, 437-448. doi: $10.3102 / 10769986031004437$

Rosseel, Y. (2012). lavaan: An R package for structural equation modeling. Journal of Statistical Software, 48(2), 1-36. doi: 10.18637/jss.v048.i02

Roth, A. J., Kornblith, A. B., Batel-Copel, L., Peabody, E., Scher, H. I., \& Holland, J. C. (1998). Rapid screening for psychologic distress in men with prostate carcinoma: A pilot study. Cancer, $\quad 82, \quad 1904-1908$ doi: 10.1002/(SICI)10970142(19980515)82:10<1904::AID-CNCR13>3.0.CO;2-X

Sawyer, A., Ayers, S., \& Field, A. P. (2010). Posttraumatic growth and adjustment among individuals with cancer or HIV/AIDS: A meta-analysis. Clinical Psychology Review, 30, 436-447. doi: 10.1016/j.cpr.2010.02.004

Shand, L. K., Cowlishaw, S., Brooker, J. E., Burney, S., \& Ricciardelli, L. A. (2015). Correlates of post-traumatic stress symptoms and growth in cancer patients: A 
systematic review and meta-analysis. Psycho-Oncology, 24, 624-634. doi: 10.1002/pon.3719

Silva, S. M., Moreira, H. C., \& Canavarro, M. C. (2012). Examining the links between perceived impact of breast cancer and psychosocial adjustment: The buffering role of posttraumatic growth. Psycho-Oncology, 21, 409-418. doi: 10.1002/pon.1913

Snowden, A., White, C. A., Christie, Z., Murray, E., McGowan, C., \& Scott, R. (2011). The clinical utility of the Distress Thermometer: A review. British Journal of Nursing, 20, 220-227. doi: 10.12968/bjon.2011.20.4.220

Stekhoven, D. J., \& Buhlmann, P. (2012). MissForest - Non-parametric missing value imputation for mixed-type data. Bioinformatics, 28, 112-118. doi: 10.1093/bioinformatics/btr597

Tedeschi, R. G., \& Calhoun, L. G. (2004). Posttraumatic growth: Conceptual foundations and empirical evidence. Psychological Inquiry, 15, 1-18. doi: 10.1207/s15327965pli1501_01

W.H.O. (2013). International perspectives on spinal cord injury. Geneva, Switzerland: WHO Press.

Widerstrom-Noga, E., Biering-Sorensen, F., Bryce, T., Cardenas, D. D., Finnerup, N. B., Jensen, M. P., . . Siddall, P. J. (2008). The International Spinal Cord Injury Pain Basic Data Set. Spinal Cord, 46, 818-823. doi: 10.1038/sc.2008.64

Zigmond, A. S., \& Snaith, R. P. (1983). The Hospital Anxiety and Depression Scale. Acta Psychiatrica Scandinavica, 67, 361-370. doi: 10.1111/j.1600-0447.1983.tb09716.x

Zoellner, T., \& Maercker, A. (2006). Posttraumatic growth in clinical psychology - A critical review and introduction of a two component model. Clinical Psychology Review, 26, 626-653. doi: 10.1016/j.cpr.2006.01.008 


\section{Tables}

Table 1

Demographic and Injury-related Characteristics of the Sample

\begin{tabular}{|c|c|c|c|}
\hline Characteristic & $n(\%)$ & $M(S D)$ & $n(\%)$ missing \\
\hline Age at discharge from first rehabilitation in years & & $53.54(17.19)$ & 0 \\
\hline Age at injury in years & & $53.09(17.17)$ & 0 \\
\hline Gender & & & 0 \\
\hline Male & $94(66.7)$ & & \\
\hline Female & $47(33.3)$ & & \\
\hline Cause of injury & & & 0 \\
\hline Traumatic & $82(58.2)$ & & \\
\hline Nontraumatic & $59(41.8)$ & & \\
\hline Marital status & & & $51(36.2)$ \\
\hline Single (never married) & $26(18.4)$ & & \\
\hline Married & 46 (32.6) & & \\
\hline Widowed & $7(5.0)$ & & \\
\hline Divorced & $10(7.1)$ & & \\
\hline Registered partnership & $1(0.7)$ & & \\
\hline Language of questionnaire & & & 0 \\
\hline German & $114(80.9)$ & & \\
\hline French & $21(14.9)$ & & \\
\hline Italian & $6(4.3)$ & & \\
\hline Time since injury in days & & $160.96(92.41)$ & 0 \\
\hline Duration of first rehabilitation in days & & $138.91(86.04)$ & 0 \\
\hline Type of injury & & & 15 (10.6) \\
\hline Incomplete paraplegia & $58(41.1)$ & & \\
\hline Complete paraplegia & $14(9.9)$ & & \\
\hline Incomplete tetraplegia & $44(31.2)$ & & \\
\hline Complete tetraplegia & $4(2.8)$ & & \\
\hline Intact & $6(4.2)$ & & \\
\hline
\end{tabular}

Note. $M$ and $S D$ rely on imputed data. 
Table 2

Distributional Properties of PTG, PTD, and of the Adjustment Indicators

\begin{tabular}{|c|c|c|c|c|c|c|c|c|c|c|c|c|}
\hline \multirow[b]{2}{*}{ Construct (measurement instrument) } & \multicolumn{2}{|l|}{$n(\%)$} & \multirow[b]{2}{*}{$M[95 \% \mathrm{CI}]$} & \multirow[b]{2}{*}{$M_{d}$} & \multirow[b]{2}{*}{$S D$} & \multicolumn{2}{|c|}{ Range } & \multirow{2}{*}{$\begin{array}{c}n(\%) \text { at } \\
\text { minimum }^{\text {b }}\end{array}$} & \multirow{2}{*}{$\begin{array}{c}n(\%) \text { at } \\
\text { maximum }^{\mathrm{c}}\end{array}$} & \multirow[b]{2}{*}{ Kurtosis } & \multirow[b]{2}{*}{ Skewness } & \multirow[b]{2}{*}{$p$} \\
\hline & Missing $^{\mathrm{a}}$ & $\alpha$ & & & & Potential & Actual & & & & & \\
\hline \multicolumn{13}{|l|}{ PTG (PTGI) } \\
\hline Total & $24(17.0)$ & .86 & $20.89[18.94,22.84]$ & 21 & 11.72 & $0-50$ & $0-47$ & $3(2.1)$ & 0 & -0.84 & 0.02 & .013 \\
\hline Relating to others & $13(9.2)$ & .52 & $4.90[4.41,5.39]$ & 5 & 2.93 & $0-10$ & $0-10$ & 15 (10.6) & $5(3.5)$ & -1.02 & -0.11 & $<.001$ \\
\hline New possibilities & $14(9.8)$ & .71 & $4.01[3.48,4.53]$ & 4 & 3.14 & $0-10$ & $0-10$ & $31(22.0)$ & $7(5.0)$ & -1.09 & 0.25 & $<.001$ \\
\hline Personal strength & $11(7.8)$ & .72 & $4.65[4.11,5.19]$ & 5 & 3.23 & $0-10$ & $0-10$ & $26(18.4)$ & $9(6.4)$ & -1.19 & -0.03 & $<.001$ \\
\hline Spiritual change & $13(9.8)$ & .79 & $2.02[1.55,2.49]$ & 0 & 2.82 & $0-10$ & $0-10$ & $78(55.3)$ & $2(1.4)$ & 0.24 & 1.21 & $<.001$ \\
\hline Appreciation of life & $9(6.4)$ & .61 & $5.30[4.80,5.81]$ & 6 & 3.02 & $0-10$ & $0-10$ & $13(9.2)$ & $12(8.5)$ & -1.01 & -0.24 & $<.001$ \\
\hline \multicolumn{13}{|l|}{ PTD (PTGI) } \\
\hline Total & $28(19.9)$ & .87 & $10.09[8.36,11.82]$ & 6 & 10.40 & $0-50$ & $0-46$ & $23(16.3)$ & 0 & 0.91 & 1.22 & $<.001$ \\
\hline Relating to others & $16(11.3)$ & .61 & $2.35[1.87,2.82]$ & 1 & 2.84 & $0-10$ & $0-10$ & $65(46.1)$ & $2(1.4)$ & -0.21 & 0.96 & $<.001$ \\
\hline New possibilities & $16(11.3)$ & .78 & $2.09[1.62,2.56]$ & 0 & 2.82 & $0-10$ & $0-10$ & $72(51.1)$ & $1(0.7)$ & 0.22 & 1.19 & $<.001$ \\
\hline Personal strength & $20(14.0)$ & .72 & $1.91[1.50,2.32]$ & 1 & 2.47 & $0-10$ & $0-9$ & 70 (49.6) & 0 & 0.65 & 1.22 & $<.001$ \\
\hline Spiritual change & $16(11.3)$ & .73 & $1.15[0.77,1.53]$ & 0 & 2.30 & $0-10$ & $0-10$ & 99 (70.2) & $3(2.1)$ & 5.02 & 2.33 & $<.001$ \\
\hline Appreciation of life & $9(6.4)$ & .73 & $2.60[2.11,3.08]$ & 1 & 2.93 & $0-10$ & $0-10$ & $53(37.6)$ & $4(2.8)$ & -0.25 & 0.94 & $<.001$ \\
\hline \multicolumn{13}{|l|}{ Adjustment indicators } \\
\hline Global distress (DT) & $2(1.4)$ & & $4.66[4.24,5.08]$ & 5 & 2.53 & $0-10$ & $0-10$ & $10(7.1)$ & $3(2.1)$ & -0.70 & 0.02 & .002 \\
\hline Depression (HADS) & $5(3.5)$ & .82 & $4.65[4.01,5.28]$ & 4 & 3.83 & $0-21$ & $0-19$ & $17(12.1)$ & 0 & 0.91 & 0.99 & $<.001$ \\
\hline Anxiety (HADS) & $2(1.4)$ & .83 & $4.81[4.16,5.45]$ & 4 & 3.87 & $0-21$ & $0-18$ & $17(12.1)$ & 0 & 0.55 & 0.89 & $<.001$ \\
\hline Life satisfaction (WHOQoL items) & $4(2.8)$ & .79 & $18.77[18.20,19.33]$ & 20 & 3.37 & $5-25$ & $8-25$ & 0 & $3(2.1)$ & 0.12 & -0.75 & $<.001$ \\
\hline Life satisfaction (SCI QoL) & $1(0.7)$ & .85 & $6.50[6.18,6.83]$ & 7 & 1.95 & $0-10$ & $0-10$ & $1(0.7)$ & $2(1.4)$ & -0.21 & -0.56 & $<.001$ \\
\hline Physical functioning (SCIM III) & $3(2.1)$ & .89 & $75.94[72.45,79.44]$ & 78 & 20.99 & $0-100$ & $14-100$ & 0 & $13(9.2)$ & 0.25 & -0.83 & $<.001$ \\
\hline Pain intensity (ISCIBPDS) & $3(2.1)$ & & $3.40[2.87,3.94]$ & 3 & 3.22 & $0-10$ & $0-10$ & $54(38.3)$ & $3(2.1)$ & -1.37 & 0.30 & $<.001$ \\
\hline
\end{tabular}

Note. Except $n(\%)$ missing, all values rely on imputed data. $p=p$-value of Shapiro-Wilk test. PTGI $=$ Posttraumatic Growth Inventory. DT $=$ Distress Thermometer. HADS $=$ Hospital Anxiety and Depression Scale. WHOQoL items $=5$ items selected from the World Health Organisation Quality of Life Assessment. SCI QoL = International Spinal Cord Injury Quality of Life Basic Data Set. SCIM III = Spinal Cord Independence Measure. ISCIBPDS = International Spinal Cord Injury Basic Pain Data Set.

${ }^{a} n(\%)$ participants with missing values in at least one of the items composing a scale. ${ }^{\mathrm{b}} n(\%)$ participants with a minimum possible raw score in a scale. ${ }^{\mathrm{c}} n(\%)$ participants with a maximum possible raw score in a scale. 


\section{Table 3}

Spearman Correlations Between PTG, PTD, and the Adjustment Indicators

\begin{tabular}{|c|c|c|c|c|c|c|c|c|c|c|c|c|c|c|c|c|c|c|}
\hline Construct (measurement instrument) & 1. & 2. & 3. & 4. & 5. & 6. & 7. & 8. & 9. & 10. & 11. & 12. & 13. & 14. & 15. & 16. & 17. & 18. \\
\hline \multicolumn{19}{|l|}{ PTG (PTGI) } \\
\hline 1. Total & & & & & & & & & & & & & & & & & & \\
\hline 2. Relating to others & $.77^{* * * *}$ & & & & & & & & & & & & & & & & & \\
\hline 3. New possiblities & $.86^{* * * *}$ & $.57^{* * * * *}$ & & & & & & & & & & & & & & & & \\
\hline 4. Personal strength & $.83^{* * * *}$ & $.54 * * *$ & $.73 * * *$ & & & & & & & & & & & & & & & \\
\hline 5. Spiritual change & $.61^{* * * *}$ & $.43^{* * * *}$ & $.42 * * *$ & $.35^{* * * *}$ & & & & & & & & & & & & & & \\
\hline 6. Appreciation of life & $.74 * * *$ & $.48^{* * * *}$ & $.54 * * *$ & $.56 * * *$ & $.29 * * *$ & & & & & & & & & & & & & \\
\hline \multicolumn{19}{|l|}{ PTD (PTGI) } \\
\hline 7. Total & $.47 * * *$ & $.37 * * *$ & $.43 * * *$ & $.35 * * *$ & $.43 * * *$ & $.31 * * *$ & & & & & & & & & & & & \\
\hline 8. Relating to others & $.46 * * *$ & $.28^{* * * *}$ & $.43 * * *$ & $.39 * * *$ & $.26 * *$ & $.37 * * *$ & $.74^{* * * *}$ & & & & & & & & & & & \\
\hline 9. New possiblities & $.30^{* * * *}$ & $.28^{* * * *}$ & $.29 * * *$ & $.20 *$ & $.38^{* * * *}$ & .11 & $.79^{* * * *}$ & $.41 * * *$ & & & & & & & & & & \\
\hline 10. Personal strength & $.31^{* * * *}$ & $.27 * *$ & $.28 * * *$ & $.19^{*}$ & $.41^{* * * *}$ & .15 & $.79^{* * * *}$ & $.54 * * *$ & $.71^{* * * *}$ & & & & & & & & & \\
\hline 11. Spiritual change & $.40^{* * * *}$ & $.26 * *$ & $.34 * * *$ & $.37 * * *$ & $.37 * * *$ & $.26 * *$ & $.55^{* * * *}$ & $.40 * * *$ & $.39 * * *$ & $.34 * * *$ & & & & & & & & \\
\hline 12. Appreciation of life & $.23^{* * *}$ & .11 & $.23 * *$ & $.18 *$ & $.37^{* * * *}$ & .12 & $.78^{* * * *}$ & $.44 * * *$ & $.58^{* * * *}$ & $.60^{* * * *}$ & $.36^{* * * *}$ & & & & & & & \\
\hline \multicolumn{19}{|l|}{ Adjustment indicators } \\
\hline 13. Global distress (DT) & $.24 * *$ & .11 & $.24 * *$ & .16 & .14 & $.30^{* * * *}$ & $.40^{* * * * *}$ & $.34 * * *$ & $.31^{* * * *}$ & $.25^{\text {*** }}$ & $.19^{*}$ & $.33^{* * * *}$ & & & & & & \\
\hline 14. Depression (HADS) & .10 & .05 & .15 & .04 & .11 & .06 & $.57 * * *$ & $.47 * * *$ & $.44 * * *$ & $.47 * * *$ & $.19^{*}$ & $.49 * * *$ & $.48 * * *$ & & & & & \\
\hline 15. Anxiety (HADS) & $.28^{* * * *}$ & $.18^{*}$ & $.27 * *$ & $.22 * *$ & $.21 *$ & $.25 * *$ & $.55^{* * * *}$ & $.46^{* * * *}$ & $.44^{* * * *}$ & $.41^{* * * *}$ & $.25 * *$ & $.43^{* * * *}$ & $.47^{* * * *}$ & $.71^{* * *}$ & & & & \\
\hline 16. Life satisfaction (WHOQoL items) & -.03 & .01 & -.10 & .00 & -.08 & .03 & $-.46^{* * * *}$ & $-.40 * * *$ & $-.40^{* * * *}$ & $-.39 * * *$ & -.06 & $-.41 * * *$ & $-38 * * *$ & $-.76 * * *$ & $-.58 * * *$ & & & \\
\hline 17. Life satisfaction (SCI QoL) & -.03 & .00 & -.11 & .01 & -.06 & .02 & $-.40^{* * * *}$ & $-.33 * * *$ & $-.36 * * *$ & $-.34 * * *$ & -.13 & $-.35^{* * * *}$ & $-.34 * * *$ & $-.70 * * *$ & $-.67 * * *$ & $.71 * * *$ & & \\
\hline 18. Physical functioning (SCIM III) & -.13 & -.03 & -.15 & -.13 & $-.19 *$ & -.01 & $-.26 * *$ & -.12 & $-.32 * * *$ & $-.26 * *$ & -.06 & $-.26 * *$ & $-.18^{*}$ & $-.26 * *$ & $-.17 *$ & $.32 * * *$ & $.18^{*}$ & \\
\hline 19. Pain intensity (ISCIBPDS) & .15 & .14 & .10 & .06 & .10 & $.18^{*}$ & $.26 * *$ & $.23 * *$ & $.21 *$ & $.18^{*}$ & .14 & .16 & $.31 * * *$ & $.30 * * *$ & $.30^{* * * *}$ & $-.35 * * *$ & $-.25 * *$ & -.11 \\
\hline
\end{tabular}

Note. All values rely on imputed data. PTGI $=$ Posttraumatic Growth Inventory. DT $=$ Distress Thermometer. HADS $=$ Hospital Anxiety and Depression Scale. WHOQoL items $=5$ items selected from the World Health Organisation Quality of Life Assessment. SCI QoL = International Spinal Cord Injury Quality of Life Basic Data Set. SCIM III = Spinal Cord Independence Measure. ISCIBPDS = International Spinal Cord Injury Basic Pain Data Set.

$* p<0.05$. ** $p<0.01$. *** $p<0.001$ 
Table 4

Hierarchical Multiple Regression Analyses Predicting the Adjustment Indicators From PTG, PTD, and Their Interaction Term

\begin{tabular}{|c|c|c|c|c|c|c|c|c|c|c|c|c|c|c|}
\hline \multirow[t]{3}{*}{ Construct (measurement instrument) } & \multicolumn{4}{|c|}{ PTG (PTGI) } & \multicolumn{4}{|c|}{ PTD (PTGI) } & \multicolumn{4}{|c|}{ PTG (PTGI) x PTD (PTGI) } & \multirow[t]{3}{*}{$\Delta R^{2}$} & \multirow[t]{3}{*}{$f^{\mathrm{b}}$} \\
\hline & & $95 \% \mathrm{CI}$ for $B$ & & & & $95 \% \mathrm{CI}$ for $B$ & & & & $95 \% \mathrm{CI}$ for $B$ & & & & \\
\hline & $L L$ & $B(S E)$ & $U L$ & $\beta$ & $L L$ & $B(S E)$ & $U L$ & $\beta$ & $L L$ & $B(S E)$ & $U L$ & $\beta$ & & \\
\hline \multicolumn{15}{|l|}{ Global distress (DT) } \\
\hline Model 1 & -.02 & $.02(.02)$ & .06 & .09 & .05 & $.09(.02)^{* * *}$ & .13 & .37 & & & & & .18 & \\
\hline Model 2 & -.02 & $.02(.02)$ & .06 & .09 & .047 & $.09(.02)^{* * * *}$ & .14 & .38 & -.00 & $-.00(.00)$ & .00 & -.03 & .00 & .00 \\
\hline \multicolumn{15}{|l|}{ Depression (HADS) } \\
\hline Model 1 & -.11 & $-.06(.03)^{*}$ & -.01 & -.20 & .19 & $.24(.03) * * *$ & .30 & .66 & & & & & .36 & \\
\hline Model 2 & -.12 & $-.07(.02) * *$ & -.03 & -.23 & .21 & $.27(.03)^{* * * *}$ & .33 & .74 & -.01 & $-.01(.002)^{*}$ & -.00 & -.18 & .03 & .04 \\
\hline \multicolumn{15}{|l|}{ Anxiety (HADS) } \\
\hline Model 1 & -.03 & $.02(.03)$ & .07 & .06 & .14 & $.19(.03)^{* * * *}$ & .25 & .52 & & & & & .30 & \\
\hline Model 2 & -.03 & $.02(.03)$ & .07 & .06 & .13 & $.19(.03)^{* * * *}$ & .26 & .52 & -.00 & $.00(.00)$ & .00 & -.00 & .00 & .00 \\
\hline \multicolumn{15}{|l|}{ Life satisfaction (WHOQoL items) } \\
\hline Model 1 & .02 & $.06(.02)^{* *}$ & .11 & .22 & -.23 & $-.18(.03) * * *$ & -.12 & -.55 & & & & & .24 & \\
\hline Model 2 & .03 & $.08(.02)^{* * *}$ & .12 & .26 & -.27 & $-.21(.03) * * *$ & -.16 & -.65 & .00 & $.01(.00)^{* *}$ & .01 & .24 & .05 & .06 \\
\hline \multicolumn{15}{|l|}{ Life satisfaction (SCI QoL) } \\
\hline Model 1 & .00 & $.03(.01) *$ & .06 & .19 & -.13 & $-.01(.02) * * *$ & -.06 & -.51 & & & & & .21 & \\
\hline Model 2 & .01 & $.04(.01)^{* * *}$ & .07 & .23 & -.15 & $-.12(.02) * * *$ & -.08 & -.62 & .00 & $.00(.00)^{* *}$ & .01 & .24 & .05 & .06 \\
\hline \multicolumn{15}{|l|}{ Physical functioning (SCIM III) } \\
\hline Model 1 & -.39 & $-.06(.17)$ & .27 & -.03 & -.83 & $-.46(.19)^{*}$ & -.09 & -.23 & & & & & .06 & \\
\hline Model 2 & -.40 & $-.07(.17)$ & .27 & -.04 & -.84 & $-.44(.20)^{*}$ & -.03 & -.22 & -.03 & $-.00(.01)$ & .02 & -.03 & .00 & .00 \\
\hline \multicolumn{15}{|l|}{ Pain intensity (ISCIBPDS) } \\
\hline Model 1 & -.04 & $.01(.03)$ & .06 & .04 & .01 & $.07(.03) *$ & .12 & .22 & & & & & .06 & \\
\hline Model 2 & -.05 & $.00(.03)$ & .05 & .00 & .04 & $.10(.03) * *$ & .16 & .32 & -.01 & $-.01(.00)^{*}$ & -.00 & -.22 & .04 & .04 \\
\hline
\end{tabular}

Note. All values rely on imputed data. $\mathrm{CI}=95 \%$ confidence interval. $L L=$ lower limit. $U L=$ upper limit. Model $1=$ regression model including only main effects. Model $2=$ hierarchical regression model including main effects (Block 1 ) and interaction term (Block 2). PTGI = Posttraumatic Growth Inventory. DT = Distress Thermometer. HADS = Hospital Anxiety and Depression Scale. WHOQoL items $=5$ items selected from the World Health Organisation Quality of Life Assessment. SCI QoL = International Spinal Cord Injury Quality of Life Basic Data Set. SCIM III = Spinal Cord Independence Measure. ISCIBPDS = International Spinal Cord Injury Basic Pain Data Set.

${ }^{a}$ PTG (PTGI) and PTD (PTGI) were centered prior to inclusion in regression models. ${ }^{b}$ Variation of Cohen's $f^{2}$ measuring local effect size.

$* p<0.05 . * * p<0.01 . * * * p<0.001$. 


\section{Figures}

a
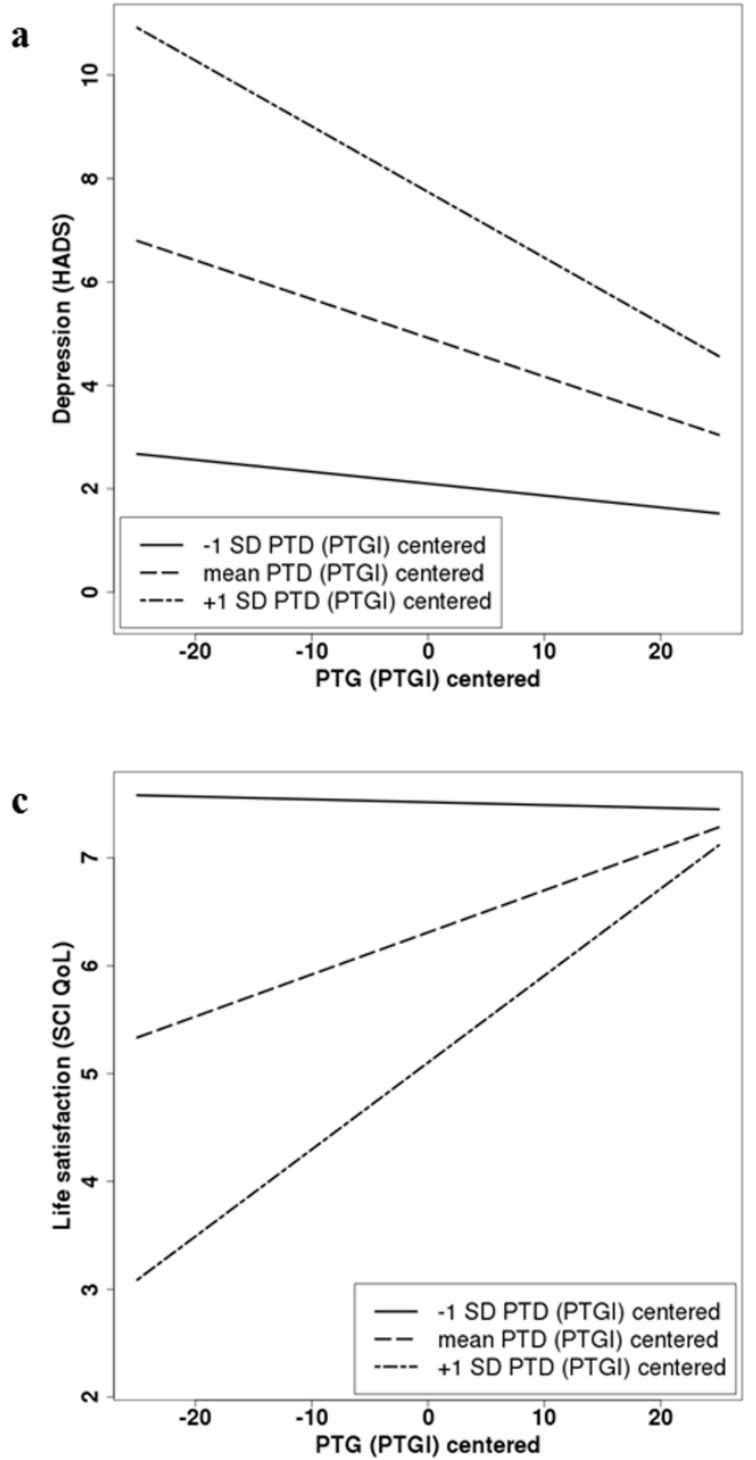

b

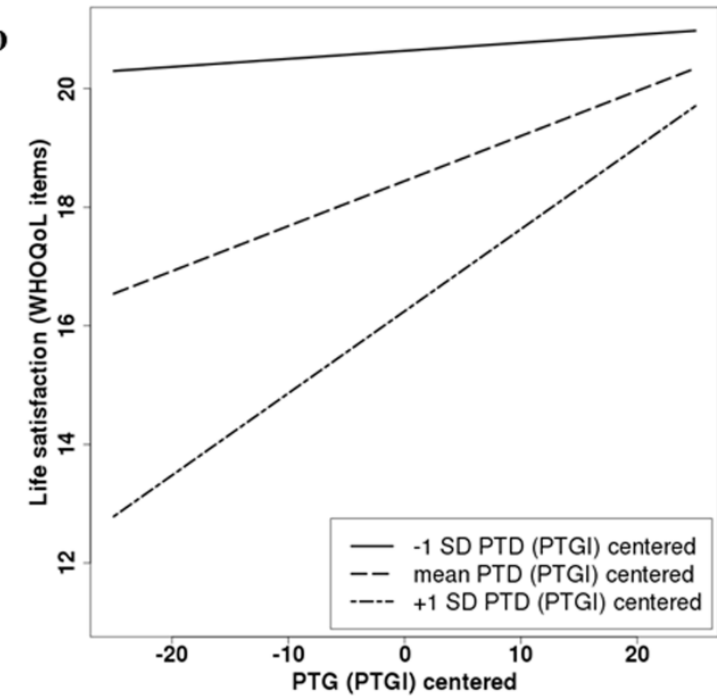

d

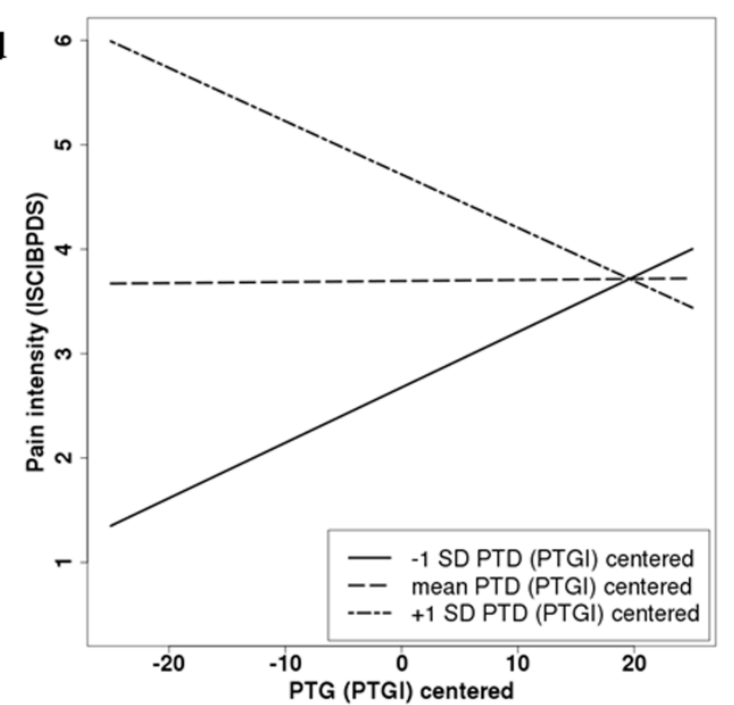

Figure 1. Plot of the interactions of centered posttraumatic growth (PTG) and posttraumatic depreciation (PTD) total scores in predicting symptoms of depression (a), life satisfaction (b and c), and pain intensity (d). 